\title{
Evidence of oxidative damage and inflammation associated with low glutathione redox status in the autism brain
}

\author{
S Rose, S Melnyk, O Pavliv, S Bai, TG Nick, RE Frye and SJ James
}

Despite increasing evidence of oxidative stress in the pathophysiology of autism, most studies have not evaluated biomarkers within specific brain regions, and the functional consequences of oxidative stress remain relatively understudied. We examined frozen samples from the cerebellum and temporal cortex (Brodmann area 22 (BA22)) from individuals with autism and unaffected controls ( $n=15$ and $n=12$ per group, respectively). Biomarkers of oxidative stress, including reduced glutathione (GSH), oxidized glutathione (GSSG) and glutathione redox/antioxidant capacity (GSH/GSSG), were measured. Biomarkers of oxidative protein damage (3-nitrotyrosine; 3-NT) and oxidative DNA damage (8-oxo-deoxyguanosine; 8-oxo-dG) were also assessed. Functional indicators of oxidative stress included relative levels of 3-chlorotyrosine (3-CT), an established biomarker of a chronic inflammatory response, and aconitase activity, a biomarker of mitochondrial superoxide production. Consistent with previous studies on plasma and immune cells, GSH and GSH/GSSG were significantly decreased in both autism cerebellum $(P<0.01)$ and BA22 $(P<0.01)$. There was a significant increase in 3-NT in the autism cerebellum and BA22 $(P<0.01)$. Similarly, 8-0xo-dG was significantly increased in autism cerebellum and BA22 $(P<0.01$ and $P=0.01$, respectively), and was inversely correlated with $G S H / G S S G$ in the cerebellum $(P<0.01)$. There was a significant increase in 3 -CT levels in both brain regions $(P<0.01)$, whereas aconitase activity was significantly decreased in autism cerebellum $(P<0.01)$, and was negatively correlated with GSH/GSSG $(P=0.01)$. Together, these results indicate that decreased GSH/GSSG redox/antioxidant capacity and increased oxidative stress in the autism brain may have functional consequence in terms of a chronic inflammatory response, increased mitochondrial superoxide production, and oxidative protein and DNA damage.

Translational Psychiatry (2012) 2, e134; doi:10.1038/tp.2012.61; published online 10 July 2012

\section{Introduction}

Autism is a complex, behaviorally defined neurodevelopmental disorder characterized by significant impairments in social interaction, verbal and non-verbal communication, and by restrictive, repetitive and stereotypic patterns of behavior. The Centers for Disease Control estimates that the current prevalence of autism spectrum disorders in the United States is 1 in 110 children. ${ }^{1}$ A number of studies have shown abnormalities in the autism brain, including the prefrontal cortex, temporal lobe, amygdala and cerebellum, as well as differences in total brain volume and growth trajectories. ${ }^{2-4}$ However, despite numerous structural and functional neuroimaging studies, as well as post-mortem investigations, the underlying neurobiological basis of autism continues to remain elusive.

Abnormalities in the cerebellum are among the most reproducibly reported alterations in the autism brain. ${ }^{2-4}$ Neuroimaging studies have reported reduced vermis volume as well as total cerebellar volume, whereas postmortem analyses have revealed a significant reduction in the number of Purkinje cells in individuals with autism. ${ }^{5-8}$
Evidence of neuroimmune involvement in the cerebellum includes the presence of activated neuroglia and elevated cytokine levels, as well as autoantibodies to cerebellar proteins. $^{9-11}$

Abnormalities in the superior temporal gyrus (STG) are thought to be relevant in autism because of its important role in processing sounds and speech development. ${ }^{12}$ The STG contains Brodmann area 22 (BA22), which in the left hemisphere corresponds to Wernicke's area, a region involved in speech processing. Neuroimaging analyses have revealed increased right STG volume in subjects with autism, consistent with a recent finding of a more rightward asymmetry of the STG in individuals with autism. ${ }^{13,14} \mathrm{~A}$ reduction in neuronal cell density and increased glial cell density was reported in a microscopic analysis of BA22 in individuals with autism. ${ }^{15}$ Neuroimmune activation in the autism STG has been reported in a single study, finding increased transcript levels of multiple immune-related genes. ${ }^{16}$

Biomarkers of oxidative stress have been reported in many neurological and psychiatric disorders, including Alzheimer's disease, ${ }^{17}$ Parkinson's disease, ${ }^{18}$ schizophrenia, ${ }^{19,20}$ bipolar

Department of Pediatrics, University of Arkansas for Medical Sciences, Arkansas Children's Hospital Research Institute, Little Rock, AR, USA

Correspondence: Dr S Rose, Department of Pediatrics, University of Arkansas for Medical Sciences, Arkansas Children's Hospital Research Institute, 13 Children's Way, Slot 512-41B, Little Rock, AR 72202, USA.

E-mail: srose@uams.edu

Keywords: aconitase; mitochondria; neuroinflammation; oxidative stress; 3-chlorotyrosine; 3-nitrotyrosine

Received 19 March 2012; revised 9 May 2012; accepted 31 May 2012 
disorder $^{21}$ and alcoholism, ${ }^{22}$ and may reflect a common underlying pathophysiological mechanism. Numerous indicators of oxidative stress have been documented previously in the blood from children with autism, including decreased antioxidant enzyme activities, elevated lipid peroxidation and accumulation of advanced glycation end products. ${ }^{23-25}$ In three independent case/control cohorts, children with autism were shown to exhibit abnormal plasma levels of metabolites in the pathway of glutathione redox metabolism. ${ }^{26-28}$ In these studies, the mean concentration of reduced glutathione (GSH), the primary intracellular antioxidant and redox buffer, was found to be significantly decreased, whereas oxidized glutathione disulfide (GSSG) was significantly increased, resulting in a decrease in the glutathione redox ratio (GSH/GSSG) in both plasma and primary immune cells from children with autism. $^{29}$ Taken together, accumulating evidence suggests that children with autism have a more oxidized extracellular (plasma) and intracellular immune cell microenvironment than age-matched unaffected control children.

Oxidative stress and damage occurs when antioxidant defense mechanisms fail to effectively counter endogenous or exogenous sources of reactive oxygen species. Glutathione is the primary antioxidant responsible for maintaining the reducing intracellular microenvironment that is essential for normal cellular function and viability. GSH/GSSG is a reliable indicator of cellular redox status, and a chronic reduction in GSH/GSSG reflects a reduced antioxidant capacity and increased vulnerability to oxidative damage. ${ }^{30}$ Recently, an increase in oxidative protein and DNA damage was associated with the decrease in intracellular and plasma GSH/ GSSG in children with autism, suggesting that the reduced antioxidant defense capacity in these children may have functional consequence in terms of overt oxidative damage. ${ }^{31}$

Although there is growing evidence that biomarkers of increased oxidative stress are present in the blood of children with autism, the presence of oxidative stress and glutathione deficit in the autism brain has remained relatively understudied. In the cerebellum of children with autism, Sajdel-Sulkowska et al. ${ }^{32,33}$ found significantly increased 3nitrotyrosine (3-NT), a marker of oxidative protein damage, while noting a trend of increased 8-oxo-deoxyguanosine (8oxo-dG), a marker of oxidative DNA damage. Another group reported a significant increase in lipid hydroperoxides in the cerebellum and temporal cortex in autism cases compared with controls. ${ }^{34}$ In addition, a greater number of cells containing lipofuscin, a matrix of lysosomal degradation products and a marker for oxidative stress, was found in BA22 in autism compared with control samples. ${ }^{15}$ Whether the pro-oxidant glutathione redox imbalance previously observed in plasma and immune cells from children with autism is also present in the autism brain has not been investigated.

We hypothesized that low glutathione redox status would be associated with elevated markers of oxidative protein and DNA damage, inflammation and mitochondrial superoxide production in two regions that have been reported to be abnormal in autism, cerebellum and BA22. To this end, we measured two stable post-translational modifications of protein tyrosine residues, 3-NT and 3-chlorotyrosine (3-CT). The 3-NT, a marker of protein oxidative damage, is formed from peroxynitrite, a highly reactive free radical generated from nitric oxide (NO) and superoxide. The tyrosine derivative, $3-C T$, is a stable marker of inflammation that is generated from hypochlorous acid, a potent chlorinating oxidant derived from myeloperoxidase in activated immune cells during an inflammatory response. ${ }^{35}$ In addition, we measured 8-oxo-dG, a commonly used biomarker for assessing oxidative DNA damage during inflammatory and pro-oxidant exposures. Finally, to assess a functional indicator of oxidative damage and mitochondrial reactive oxygen species production, the activity of aconitase, a redox-sensitive enzyme in the tricarboxylic acid cycle, was measured. Aconitase is highly sensitive to inactivation by superoxide because of its labile iron atom and its proximity to the superoxide-generating electron transport chain. ${ }^{36}$ As $85 \%$ of aconitase in the brain is the mitochondrial isozyme, a decrease in brain aconitase activity is considered a sensitive indicator of excess mitochondrial superoxide production. ${ }^{37-39}$

Taken together, the findings of this study support our hypothesis and a role for glutathione redox imbalance and oxidative stress in the neuropathology of autism. Further, this study provides new evidence that mitochondrial superoxide production may be elevated in certain brain regions and that a neuroinflammatory process may promote oxidative stress and damage in affected cells.

\section{Materials and methods}

Post-mortem brain samples. Frozen post-mortem tissues were obtained from the NICHD Brain and Tissue Bank for Developmental Disorders at the University of Maryland, Baltimore, MD, USA, and from the Autism Tissue Program at the Harvard Brain Tissue Resource Center, Belmont, MA, USA. A total of 15 autism and 15 control tissues from cerebellar cortex, and 12 autism and 12 control tissues from BA22 were evaluated. Diagnosis of autism was confirmed by the Autism Tissue Program, using the Autistic Diagnostic Interview Revised. Autism and control groups were perfectly matched for gender, and were matched as closely as possible for post-mortem interval, age, race and cause of death. The phenotypic description of the autism case and control tissues from the cerebellum and BA22 are presented in Supplementary Tables 1 and 2, respectively. Although behavioral scores from the Autistic Diagnostic Interview Revised were available on a few samples through the Autism Tissue Program database, there were insufficient numbers to perform valid correlations between biomarkers and behavior.

Aconitase activity. To evaluate a functional consequence of oxidative stress, the activity of the redox-sensitive enzyme, aconitase, was measured using the Aconitase Assay Kit (Cayman Chemical, Ann Arbor, MI, USA) following the manufacturer's instructions. The assay is based on the conversion of citrate to isocitrate, to $\alpha$-ketoglutarate, which results in the production of NADPH. The assay measures the increase in absorbance monitored at $340 \mathrm{~nm}$ associated with the formation of $\mathrm{NADPH}$, which is proportional to the aconitase activity. Approximately $100 \mathrm{mg}$ of frozen tissue was minced in $500 \mu \mathrm{l}$ ice-cold homogenization buffer and homogenized in a Dounce homogenizer. Homogenates were 
centrifuged at $800 \mathrm{~g}$ for $10 \mathrm{~min}$ at $4{ }^{\circ} \mathrm{C}$, and the supernatant was sonicated on ice for $20 \mathrm{~s}$ before protein quantification (BCA protein assay, Pierce, Rockford, IL, USA). Samples were diluted to $0.5 \mathrm{mg} \mathrm{ml}^{-1}$ in assay buffer immediately before the assay.

High-performance liquid chromatography quantification of GSH/GSSG, 3-NT and 3-CT. To evaluate biomarkers of oxidative stress and damage by high-performance liquid chromatography (HPLC), approximately $200 \mathrm{mg}$ of tissue was minced and homogenized in $500 \mu \mathrm{l}$ of ice-cold phosphatebuffered saline. To precipitate proteins, $150 \mu \mathrm{l}$ of $10 \%$ metaphosphoric acid was added to $100 \mu$ of tissue homogenate and incubated for $30 \mathrm{~min}$ on ice. The samples were then centrifuged at $18000 \mathrm{~g}$ at $4{ }^{\circ} \mathrm{C}$ for $15 \mathrm{~min}$, and $20 \mu \mathrm{l}$ of the resulting supernatants was injected into the HPLC column for metabolite quantification, while the pellet was used for protein analysis using the BCA protein assay. The methodological details for HPLC elution and electrochemical detection of free unbound GSH, GSSG, 3-NT and 3-CT have been described previously. ${ }^{40,41}$ The results are expressed as per $\mathrm{mg}$ protein.

HPLC/mass spectrometry quantification of 8-oxodG. DNA was extracted from brain tissues using standard phenol chloroform methodology for which the methodological details have been published previously. ${ }^{31}$ Briefly, to $\sim 1 \mu \mathrm{g}$ DNA, RNase A (Sigma, St Louis, MO, USA) was added to a final concentration of $0.02 \mathrm{mg} \mathrm{ml}^{-1}$ and incubated at $37^{\circ} \mathrm{C}$ for $15 \mathrm{~min}$. The purified DNA was digested into component nucleotides using nuclease $P_{1}$, snake venom phosphodiesterase and alkaline phosphatase as previously described in detail. ${ }^{42}$ Quantification of 8-oxo-dG in DNA was performed using mass spectrometry/liquid chromatography on a LCQ Advantage MAX system (Thermo Electron Corporation, Waltham, MA, USA) and expressed as pmol per mg DNA. ${ }^{43}$

Statistical analyses. Within each region, biomarkers were evaluated using a multiple linear regression to test the group difference between cases and controls. Age, sex and postmortem interval were included as covariates, and controlled for in the regression analysis. The means, s.d. and associated $P$-values for case and control samples are reported. Spearman's correlation coefficients were computed to determine inter-metabolite correlations. All data were analyzed using SAS 9.2 (SAS Institute, Cary, NC, USA). All statistical tests used a significance level of 0.05 .

\section{Results}

Demographics of tissue donors. There were no differences in mean age, gender or post-mortem interval between the autism and control groups for either cerebellum or BA22. Race and cause of death were matched as closely as possible between available case and control samples; however, in a few subjects, the race and cause of death were unknown (Supplementary Tables 1 and 2).

GSH, GSSG, 3-NT, 3-CT and 8-oxo-dG. Figure 1 presents the relative concentrations of GSH, GSSG, 3-NT, 3-CT and
8-oxo-dG in the autism and control cerebellum, and the BA22 samples. All measured metabolites were significantly altered in autism compared with matched controls in both brain regions analyzed. GSH was decreased in autism cerebellum by $43 \%$ compared with control cerebellum $(P<0.01)$. Similarly, in BA22, GSH was $32 \%$ decreased in autism cases compared with controls $(P<0.01)$. The concentration of GSSG was $18 \%$ higher $(P=0.02)$ and the resulting $\mathrm{GSH} /$ GSSG redox ratio was $52 \%$ lower $(P<0.01)$ in the cerebellum from autism cases compared with controls. In BA22, the autism samples exhibited $19 \%$ higher concentration of GSSG $(P<0.01)$ and $43 \%$ lower $\mathrm{GSH} /$ GSSG $(P<0.001)$ redox ratio compared with controls. The concentration of 3-NT was elevated by 42 and $72 \%$ in autism cerebellum $(P<0.01)$ and BA22 $(P<0.01)$, respectively, compared with control samples. The level of 3-CT was significantly increased by 95 and $38 \%$ in autism cerebellum $(P<0.01)$ and BA22 $(P<0.01)$, respectively, compared with control samples. The mean concentration of 8 -oxo-dG was increased $27 \%$ in cerebellum $(P<0.01)$ and $21 \%$ in BA22 $(P=0.01)$ in autism compared with control tissues. Age was not significantly correlated with any of the measured biomarkers; however, post-mortem interval was negatively associated with GSSG in the combined case/control cohort of samples from BA22 $(r=-0.57 ; P=0.004)$ and in the autism BA22 samples $(r=-0.63 ; P=0.03)$.

Aconitase activity. Figure 2 a presents the aconitase activity (nmol min ${ }^{-1} \mathrm{ml}^{-1}$ ) measured in both cerebellum and BA22 autism and control samples. The mean aconitase activity in the cerebellum from the autism cases was significantly lower than in the cerebellum tissues from controls (45.3\%; $P<0.01)$. There was a trend of decreased aconitase activity in BA22 from the autism cases compared with controls; however, it failed to reach statistical significance $(P=0.1)$. A positive association between aconitase activity and GSH/GSSG was found in both cerebellum $(P=0.01)$ and BA22 $(P=0.03)$ in our combined case and control cohort (correlation coefficients $=0.46$ and 0.45 , respectively). Figures $2 b$ and $c$ illustrate this relationship and demonstrate that the autism cases are clustered in the lower left quadrant of the graphs with decreased GSH/GSSG and aconitase activity relative to controls.

8-oxo-dG is associated with GSH/GSSG in the cerebellum. Figure 3 illustrates the significant negative association found between 8-oxo-dG and GSH/GSSG in the cerebellum in the combined case and control cohort (correlation coefficient $=-0.78 ; P<0.0001$ ). Although the association was not significant within the autism cohort, the clustering of the autism cases in the bottom right quadrant with significantly higher 8-oxo-dG levels and lower GSH/GSSG is apparent.

\section{Discussion}

In the present investigation, we demonstrate for the first time that the decreased glutathione-mediated redox/antioxidant capacity previously observed in plasma and immune cells from children with autism is also significantly decreased in two 
a

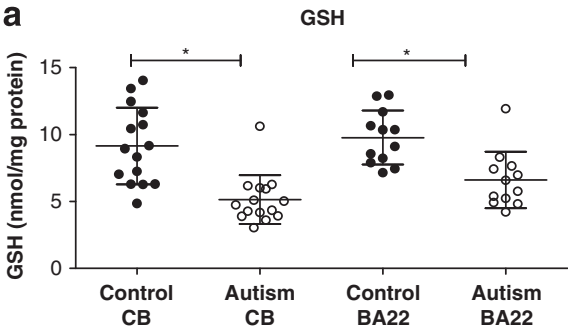

C

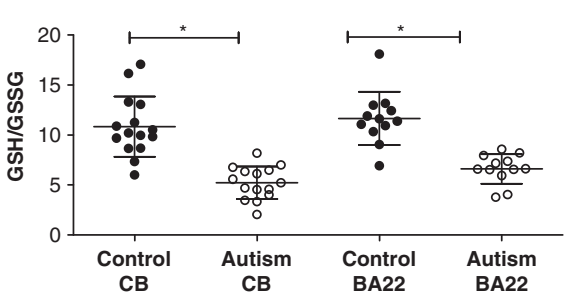

e

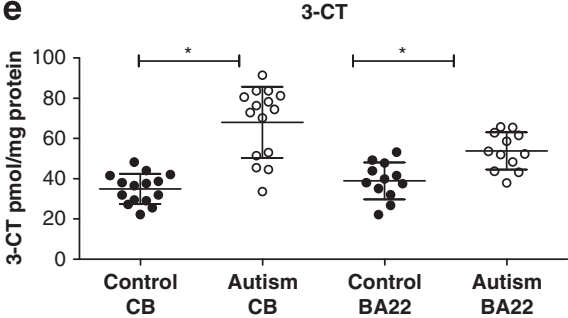

b

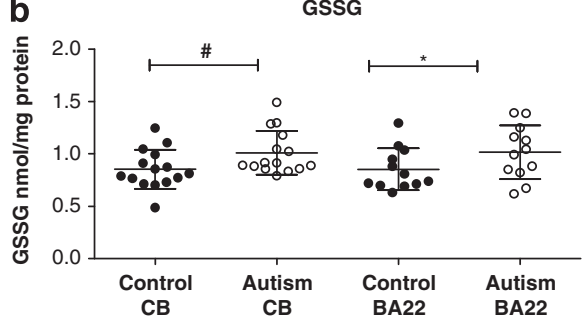

d

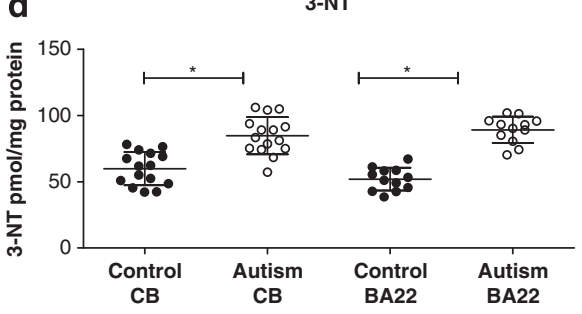

f

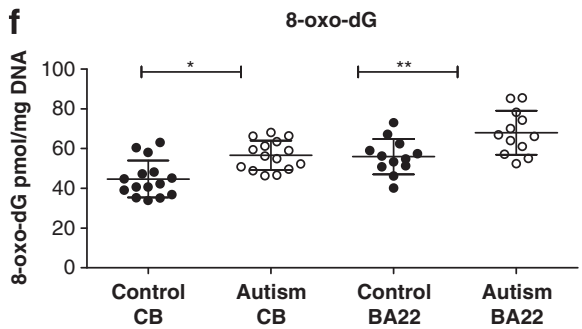

Figure 1 Glutathione redox imbalance and increased biomarkers of oxidative stress in autism cerebellum (CB) and Brodmann area 22 (BA22). High-performance liquid chromatography (HPLC) and HPLC/mass spectrometry were used to measure biomarkers in autism and control tissue samples from CB and BA22 ( $n=15$ and 12 cases and controls/group, respectively), and normalized for protein content. The concentrations of reduced glutathione (GSH; $\mathbf{a})$, oxidized glutathione (GSSG; $\mathbf{b}$ ), glutathione redox/ antioxidant capacity (GSH/GSSG; c), 3-nitrotyrosine (3-NT; d), 3-chlorotyrosine (3-CT; e), 8-oxo-deoxyguanosine (8-oxo-dG; f) are presented as mean \pm s.d. ${ }^{*} P<0.01$; ${ }^{\star *} P=0.01 ;{ }^{\#} P=0.02$.

brain regions previously shown to be affected in autism, the cerebellum and BA22. Our findings also confirm previous preliminary reports that markers of oxidative damage (3-NT and 8-oxo-dG) are increased in these two brain regions in individuals with autism. ${ }^{32,44}$ We further extend these findings by examining a larger sample of carefully selected tissues for multiple markers of oxidative protein/DNA damage (3-NT, 8-oxo-dG), as well as functional biomarkers of inflammation (3-CT) and mitochondrial superoxide production (aconitase activity).

A relative decrease in aconitase activity in the autism cerebellum is an important new finding, suggesting a functional consequence of oxidative stress in this region. In brain tissue, aconitase is located primarily in the mitochondria where it functions as an enzyme in the tricarboxylic acid cycle. ${ }^{37}$ Mitochondrial aconitase is highly sensitive to oxidative inactivation by superoxide radicals that are produced in close proximity by the electron transport chain (ETC). Thus, in addition to being a marker of oxidative protein damage, a decrease in aconitase activity is considered to be a sensitive indicator of elevated mitochondrial superoxide production. ${ }^{45,46}$

The labile iron-sulfur (Fe-S) cluster present in the active site of aconitase is a major target of excessive mitochondrial superoxide. In the presence of sufficient reducing agents, such as GSH or NADPH, aconitase can be restored to its active form; ${ }^{47}$ however, in the autism cerebellum, the observed decrease in GSH concentration relative to controls indicates a chronic deficit of reducing equivalents in this region. As depicted in Figure 4, unscavenged superoxide inactivates aconitase by displacing $\mathrm{Fe}^{+2}$ from the $\mathrm{Fe}-\mathrm{S}$ cluster, which then promotes the formation of the damaging hydroxyl radical via reaction with $\mathrm{H}_{2} \mathrm{O}_{2}$ and Fenton chemistry. A fragile redox state within the mitochondria of individuals with autism has been previously reported, ${ }^{48}$ and may reflect a selfamplifying cycle of antioxidant depletion and aconitase inactivation. Several enzymes involved in ATP production contain Fe-S clusters in the active site and are subject to similar inactivation by superoxide, including oxoglutarate dehydrogenase of the tricarboxylic acid cycle, as well as ETC complexes I-III. ${ }^{49}$ A deficit in the tricarboxylic acid cycle and ETC function under conditions of excessive superoxide production in the brain would be expected to result in a reduced ability to maintain adequate levels of ATP required for normal neuronal and synaptic functioning.

Aconitase inactivation and oxidative stress have been noted in other neuropsychiatric and neurodegenerative disorders with known mitochondrial involvement, including schizophrenia, ${ }^{50,51}$ Huntington's disease ${ }^{52}$ and Parkinson's disease. ${ }^{53,54}$ One study of mitochondrial dysfunction in the autism brain found decreased protein levels of multiple ETC complexes in the cerebellum, frontal and temporal cortex. ${ }^{34}$ There is mounting evidence that mitochondrial dysfunction may be present in a significant subset of children with autism 

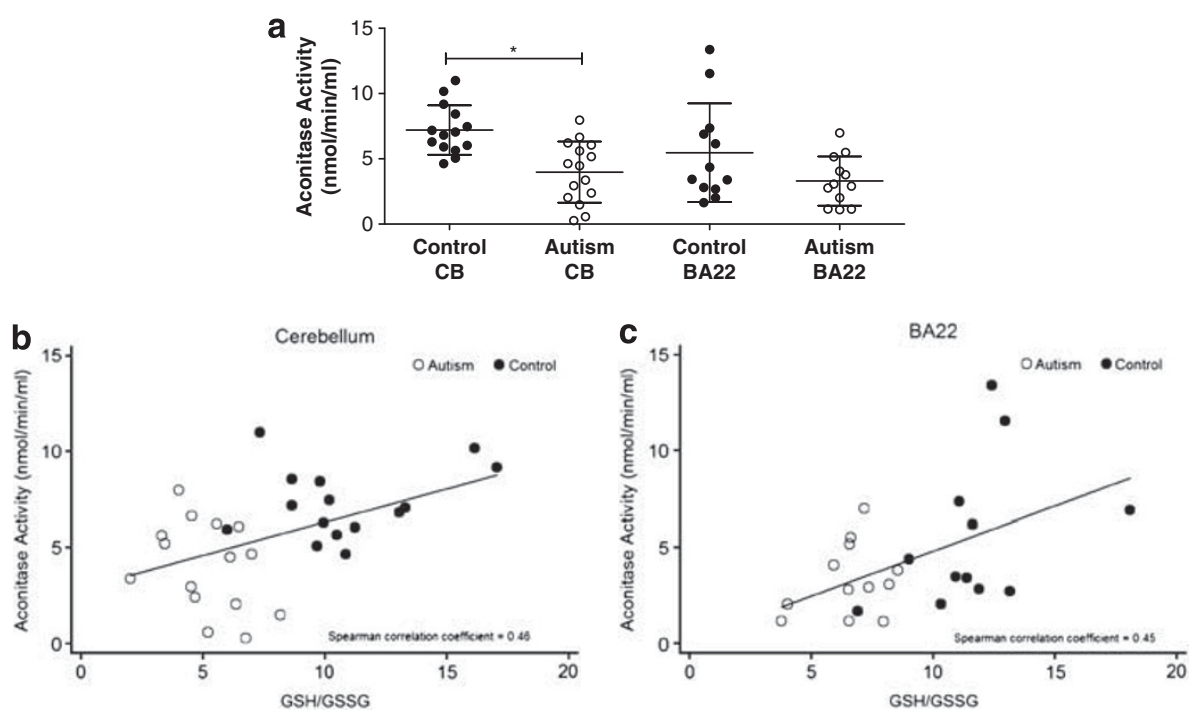

Figure 2 Aconitase activity is decreased in autism cerebellum (CB) and associated with glutathione redox/antioxidant capacity (GSH/GSSG) in control and autism CB, and Brodmann area 22 (BA22). (a) Aconitase activity was measured in frozen post-mortem autism and control tissue samples from CB and BA22 ( $n=15$ and 12 cases and controls/group, respectively), and normalized for protein content. Data are presented as mean \pm s.d. Aconitase activity was significantly decreased in autism $\mathrm{CB}(3.99 \pm 2.34)$ compared with control CB $(7.29 \pm 1.85)$. The difference in aconitase activity between autism BA22 $(3.30 \pm 1.88)$ and control BA22 (5.47 \pm 3.78$)$ did not reach significance. (b) In the combined case and control cohort of samples from the $\mathrm{CB}$, aconitase activity was significantly associated with GSH/GSSG $(P=0.01)$. (c) Within BA22 in the combined case and control samples, aconitase activity was similarly significantly associated with GSH/GSSG $(P=0.03)$. Although the significance of the correlations does not hold within the autism samples, the sample-specific clustering of case values (in open circles) in the bottom left quadrant of each graph is apparent. ${ }^{*} P<0.01$.

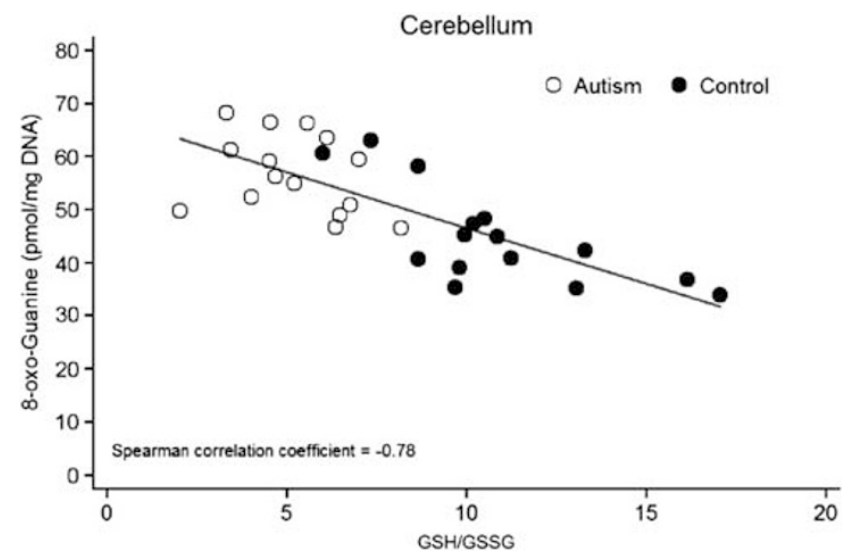

Figure 3 The 8-oxo-deoxyguanosine (8-0xo-dG) is associated with glutathione redox/antioxidant capacity (GSH/GGSG) in the cerebellum. In the combined case and control cohort of samples from the cerebellum, 8-oxo-dG was significantly associated with GSH/GSSG $(P<.0001)$. Although the significance of the correlations does not hold within the autism samples, the sample-specific clustering of case values (in open circles) in the bottom left quadrant of each graph is apparent.

and contribute to the multisystem abnormalities seen in some autistic children. ${ }^{55-57}$ The significant decrease in aconitase activity warrants continued investigation into interactions between mitochondrial dysfunction, superoxide production and altered ETC complex activity in autism.

The oxidized protein tyrosine derivative, 3-NT, provides a stable biochemical footprint of oxidative protein damage and has been found to be elevated in plasma of children with autism in a previous study. ${ }^{31}$ Elevated levels of 3-NT have been described in a number of diseases with an oxidative stress pathology, including alcoholism, smoking, diabetes, atherosclerosis and cystic fibrosis. ${ }^{58}$ The tyrosine derivative, 3-NT, is formed primarily from peroxynitrite, a damaging free radical generated from superoxide and NO. Thus, the significant increase in levels of 3-NT observed in the autism cerebellum and BA22 was not unexpected and is consistent with elevated superoxide production and aconitase inactivation.

In addition to being a classic marker of oxidative protein damage, elevated levels of 3-NT indicates elevated NO production. Excessive NO competes with the antioxidants, MnSOD and GSH, for superoxide and promotes the generation of peroxynitrite. ${ }^{59} \mathrm{NO}$ can reversibly inhibit mitochondrial respiration at complex IV, whereas the more damaging peroxynitrite can permanently inactivate complexes I, III and $\mathrm{V}^{60}$ In a previous study, we demonstrated the increased sensitivity of autism lymphoblastoid cells to acute NO-induced mitochondrial membrane depolarization, and others have reported elevated plasma and red blood cells levels of nitrites in children with autism. ${ }^{24,48,61,62}$ Neuroglial cells express iNOS (inducible NO synthase) and produce high quantities of NO when activated by cytokines. ${ }^{63,64}$ Interestingly, the presence of proinflammatory cytokines and activated neuroglia have been reported in the autism cerebellum among other regions, ${ }^{9}$ suggesting that activated neuroglia produce excess $\mathrm{NO}$ and may contribute to the peroxynitrite formation and increased 3-NT protein damage observed in the present study.

A significantly elevated level of 3-CT in the autism cerebellum and BA22 is a novel finding indicative of a chronic neuroinflammatory state in these regions. Activated phagocytic cells produce hypochlorous acid, the product of myeloperoxidase (MPO) activity that is stimulated during 


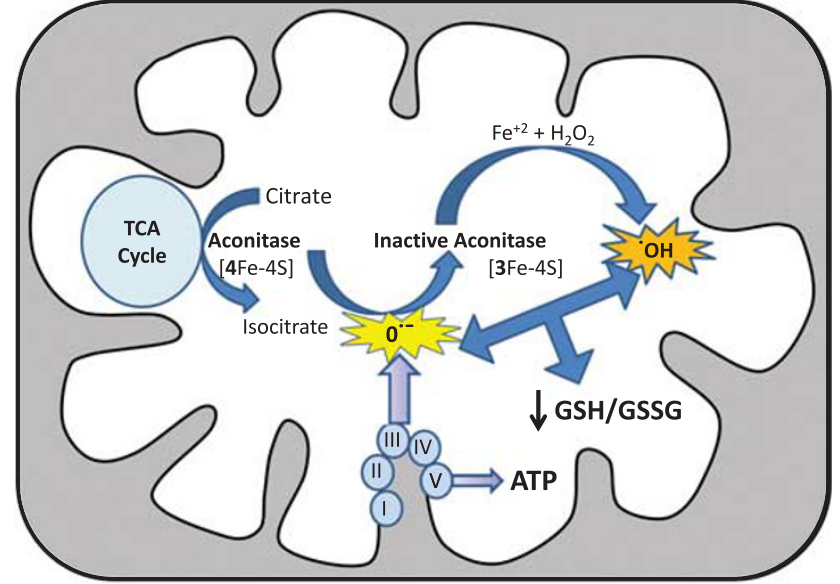

Figure 4 Mechanism of mitochondrial aconitase inactivation. Mitochondrial aconitase is a tricarboxylic acid (TCA) cycle enzyme that catalyzes the conversion of citrate to isocitrate. It contains an iron-sulfur cluster ([4Fe-4S]) in its active site that is inactivated by superoxide $\left(\mathrm{O}^{-}\right)$produced in close proximity as a byproduct of the electron transport chain. This results in the release of a Fe${ }^{+2}$ and a molecule of hydrogen peroxide $\left(\mathrm{H}_{2} \mathrm{O}_{2}\right)$, which, through the Fenton reaction, can react to produce a hydroxyl radical $\left(\bullet^{\bullet} \mathrm{OH}\right)$. The glutathione redox capacity (GSH/GSSG) will decrease as a result of the elevated free-radical production and will allow more superoxide inactivation of aconitase, creating a self-amplifying cycle if left unresolved.

immune activation, resulting in the $3-\mathrm{CT}$ derivative. ${ }^{35}$ Elevated expression of MPO has previously been demonstrated in chronic neurological disease states, such as Alzheimer's disease, ${ }^{65}$ Parkinson's disease ${ }^{66}$ and multiple sclerosis. ${ }^{67}$ The observed increase in 3-CT in the autism cerebellum and BA22 samples is the first indication of elevated MPO expression in the autism brain, and supports previous reports of microglial activation and inflammatory cytokines in autism cerebellum. ${ }^{9}$ The role of inflammation and microglial activation in the neuropathology of autism warrants further investigation and confirmation.

In addition to markers of protein oxidative damage, 8-oxo$\mathrm{dG}$, a marker of DNA oxidative damage, was significantly elevated in both cerebellum and BA22 from the autism cases relative to controls. The 8 -oxo-dG adduct in the mitochondrial and nuclear DNA is a pre-mutagenic lesion formed primarily by an attack by the hydroxyl radical $\left({ }^{\bullet} \mathrm{OH}\right)$. It has been associated with oxidative DNA damage in conditions such as aging, cancer and pro-oxidant environmental exposures. ${ }^{68}$ In the cerebellum, 8-oxo-dG was negatively associated with GSH/GSSG in the combined case and control cohort (Figure 3); however, this association failed to reach significance in the BA22 region. The superoxide-mediated release of $\mathrm{Fe}^{+2}$ associated with mitochondrial aconitase inactivation has been shown to be a significant source of $\mathrm{OH}$ radical formation through Fenton chemistry. ${ }^{47}$ Taken together, these data suggest the reduced GSH/GSSG antioxidant capacity is insufficient to counter excessive ${ }^{\circ} \mathrm{OH}$ production, and that unopposed ${ }^{\bullet} \mathrm{OH}$ can reach the nucleus to create the oxidative DNA adduct, 8-oxo-dG.

The hypothesized interactions between each of the measured biomarkers of oxidative stress and damage, mitochondrial dysfunction and inflammation are diagrammed

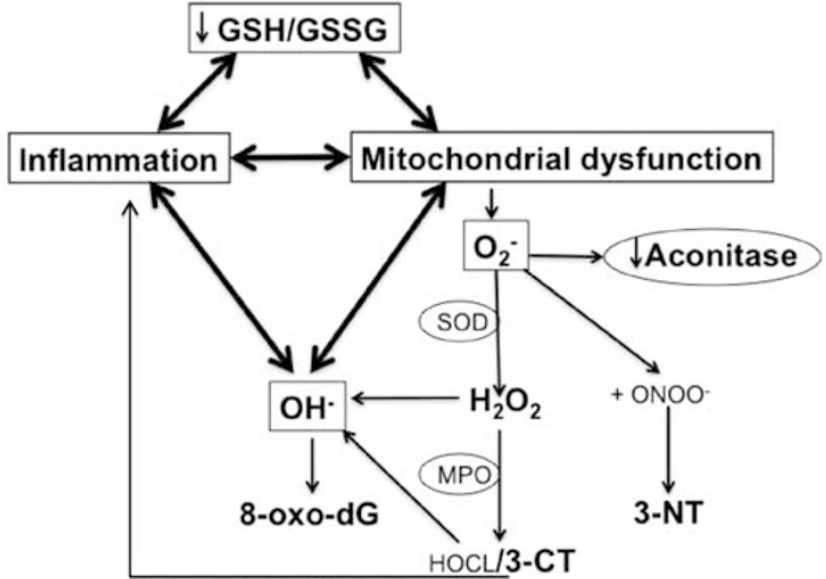

Figure 5 Proposed interactions between measured biomarkers and oxidative stress. Elevated superoxide generated from dysfunctional mitochondria promotes the formation of excess $\mathrm{H}_{2} \mathrm{O}_{2}$, the substrate for myeloperoxidase (MPO)-mediated hypochlorous acid $(\mathrm{HOCl})$ synthesis and the generation of the inflammatory biomarker, 3-chlorotyrosine (3-CT). An elevation in nitric oxide (NO) combined with elevated superoxide levels results in the formation of the peroxynitrite radical and the protein oxidative damage biomarker, 3-nitrotyrosine (3-NT). The hydroxyl radical is generated by both aconitase inactivation and MPO, and promotes the formation of 8-oxo-deoxyguanosine (8-oxo-dG). Chronic elevation of these free radicals will deplete the glutathione redox/antioxidant capacity (GSH/GSSG), allowing unopposed free-radical generation and a self-perpetuating cycle, leading to chronic oxidative stress.

in Figure 5. Elevated superoxide generated from dysfunctional mitochondria promotes the formation of excessive $\mathrm{H}_{2} \mathrm{O}_{2}$, the substrate for MPO-mediated hypochlorous acid synthesis and the generation of the inflammatory biomarker, 3-CT. Elevated superoxide can combine with NO, resulting in the formation of the peroxynitrite radical and the protein oxidative damage biomarker, 3-NT. The hydroxyl radical is generated by both aconitase inactivation and MPO activity, and promotes the formation of 8-oxo-dG. Chronic elevation of these free radicals will deplete GSH/GSSG redox/antioxidant capacity, allowing unopposed free-radical generation and a self-perpetuating cycle, leading to chronic oxidative stress and damage. The lack of a correlation between age and the biomarkers suggests that oxidative stress is a chronic condition in autism, because the same pattern of elevated biomarkers is seen over such a wide age range.

In summary, we show for the first time that peripheral markers of oxidative stress and damage previously observed in plasma and immune cells are similarly elevated in two affected brain regions in autism, cerebellum and BA22. Together, these observations suggest that a pro-oxidant environment and oxidative stress are pervasive and systemic in individuals with autism. The negative association between GSH/GSSG, and oxidative protein and DNA damage suggest that decreased glutathione redox capacity in the autism brain may have functional consequence in terms of increased mitochondrial superoxide production and a chronic inflammatory state. Nonetheless, because autism is influenced by multiple interacting genetic and environmental factors that are case-specific and inherent limitations in post-mortem brain, these observations will require confirmation in subsequent studies. 


\section{Conflict of interest}

The authors declare no conflict of interest.

Acknowledgements. We would like to thank the families of individuals with autism for the thoughtful donation of post-mortem tissues to the Autism Tissue Program at the Harvard Brain Tissue Resource Center, Belmont, MA,USA, and the NICHD Brain and Tissue Bank for Developmental Disorders at the University of Maryland, Baltimore, MD, USA. This work was supported, in part, by the National Institute of Child Health and Development (RO1 HD051873 to SJJ) and the Jane Botsford Johnson Foundation.

1. CDC. Prevalence of autism spectrum disorders - Autism and Developmental Disabilities Monitoring Network, United States, 2006. MMWR Surveill Summ 2009; 58: 1-20.

2. Amaral DG, Schumann CM, Nordahl CW. Neuroanatomy of autism. Trends Neurosci2008; 31: $137-145$

3. Brambilla P, Hardan A, di Nemi SU, Perez J, Soares JC, Barale F. Brain anatomy and development in autism: review of structural MRI studies. Brain Res Bull 2003; 61: 557-569.

4. Pardo CA, Eberhart CG. The neurobiology of autism. Brain Pathol 2007; 17: 434-447.

5. Whitney ER, Kemper TL, Bauman ML, Rosene DL, Blatt GJ. Cerebellar Purkinje cells are reduced in a subpopulation of autistic brains: a stereological experiment using calbindinD28k. Cerebellum 2008; 7: 406-416.

6. Scott JA, Schumann CM, Goodlin-Jones BL, Amaral DG. A comprehensive volumetric analysis of the cerebellum in children and adolescents with autism spectrum disorder. Autism Res 2009; 2: 246-257.

7. Courchesne E, Karns CM, Davis HR, Ziccardi R, Carper RA, Tigue ZD et al. Unusual brain growth patterns in early life in patients with autistic disorder: an MRI study. Neurology 2001; 57: $245-254$

8. Kemper TL, Bauman ML. The contribution of neuropathologic studies to the understanding of autism. Neurol Clin 1993; 11: 175-187.

9. Vargas DL, Nascimbene C, Krishnan C, Zimmerman AW, Pardo CA. Neuroglial activation and neuroinflammation in the brain of patients with autism. Ann Neurol 2005; 57: 67-81.

10. Singer HS, Morris CM, Williams PN, Yoon DY, Hong JJ, Zimmerman AW. Antibrain antibodies in children with autism and their unaffected siblings. J Neuroimmunol 2006; 178 149-155.

11. Goines $P$, Haapanen L, Boyce R, Duncanson P, Braunschweig D, Delwiche L et al. Autoantibodies to cerebellum in children with autism associate with behavior. Brain Behav Immun 2011; 25: 514-523.

12. Bigler ED, Mortensen S, Neeley ES, Ozonoff S, Krasny L, Johnson M et al. Superior temporal gyrus, language function, and autism. Dev Neuropsychol 2007; 31: 217-238.

13. Gage NM, Juranek J, Filipek PA, Osann K, Flodman P, Isenberg AL et al. Rightward hemispheric asymmetries in auditory language cortex in children with autistic disorder: an MRI investigation. J Neurodev Disord 2009; 1: 205-214.

14. Jou RJ, Minshew NJ, Keshavan MS, Vitale MP, Hardan AY. Enlarged right superior temporal gyrus in children and adolescents with autism. Brain Res 2010; 1360: 205-212

15. Lopez-Hurtado E, Prieto JJ. A microscopic study of language-related cortex in autism. Am Biochem Biotechnol 2008; 4: 130-145

16. Garbett K, Ebert PJ, Mitchell A, Lintas C, Manzi B, Mirnics K et al. Immune transcriptome alterations in the temporal cortex of subjects with autism. Neurobiol Dis 2008; 30:303-311.

17. Butterfield DA, Drake J, Pocernich C, Castegna A. Evidence of oxidative damage in Alzheimer's disease brain: central role for amyloid beta-peptide. Trends Mol Med 2001; 7 548-554

18. Martin HL, Teismann P. Glutathione-a review on its role and significance in Parkinson's disease. FASEB J 2009; 23: 3263-3272.

19. Nishioka N, Arnold SE. Evidence for oxidative DNA damage in the hippocampus of elderly patients with chronic schizophrenia. Am J Geriatr Psychiatry 2004; 12: 167-175.

20. Yao JK, Leonard S, Reddy R. Altered glutathione redox state in schizophrenia. Dis Markers 2006: 22: 83-93.

21. Andreazza AC, Kauer-Sant'anna M, Frey BN, Bond DJ, Kapczinski F, Young LT et al. Oxidative stress markers in bipolar disorder: a meta-analysis. J Affect Disord 2008; 111 135-144.

22. Nordmann R. Oxidative stress from alcohol in the brain. Alcohol Alcohol Suppl 1987; 1 : 75-82.

23. Chauhan A, Chauhan V, Brown WT, Cohen I. Oxidative stress in autism: increased lipid peroxidation and reduced serum levels of ceruloplasmin and transferrin-the antioxidan proteins. Life Sci 2004; 75: 2539-2549.

24. Sogut S, Zoroglu SS, Ozyurt H, Yilmaz HR, Ozugurlu F, Sivasli E et al. Changes in nitric oxide levels and antioxidant enzyme activities may have a role in the pathophysiological mechanisms involved in autism. Clin Chim Acta 2003; 331: 111-117.

25. Boso M, Emanuele E, Minoretti P, Arra M, Politi P, Ucelli di Nemi S et al. Alterations of circulating endogenous secretory RAGE and S100A9 levels indicating dysfunction of the AGE-RAGE axis in autism. Neurosci Lett 2006; 410: 169-173.
26. James SJ, Cutler P, Melnyk S, Jernigan S, Janak L, Gaylor DW et al. Metabolic biomarkers of increased oxidative stress and impaired methylation capacity in children with autism. Am J Clin Nutr 2004; 80: 1611-1617.

27. James SJ, Melnyk S, Fuchs G, Reid T, Jernigan S, Pavliv $O$ et al. Efficacy of methylcobalamin and folinic acid treatment on glutathione redox status in children with autism. Am J Clin Nutr 2009; 89: 425-430.

28. James SJ, Melnyk S, Jernigan S, Cleves MA, Halsted CH, Wong DH et al. Metabolic endophenotype and related genotypes are associated with oxidative stress in children with autism. Am J Med Genet B Neuropsychiatr Genet 2006; 141B: 947-956.

29. Rose S, Melnyk S, Trusty TA, Pavliv O, Seidel L, Li J et al. Intracellular and extracellular redox status and free radical generation in primary immune cells from children with autism. Autism Res Treatment 2011; 2012.

30. Jones DP. Extracellular redox state: refining the definition of oxidative stress in aging. Rejuvenation Res 2006; 9: 169-181.

31. Melnyk S, Fuchs GJ, Schulz E, Lopez M, Kahler SG, Fussell JJ et al. Metabolic imbalance associated with methylation dysregulation and oxidative damage in children with autism. J Autism Dev Disord 2012; 42: 367-377.

32. Sajdel-Sulkowska EM, Xu M, Koibuchi N. Increase in cerebellar neurotrophin-3 and oxidative stress markers in autism. Cerebellum 2009; 8: 366-372

33. Sajdel-Sulkowska EM, Xu M, McGinnis W, Koibuchi N. Brain region-specific changes in oxidative stress and neurotrophin levels in autism spectrum disorders (ASD). Cerebellum 2011; 10: 43-48

34. Chauhan A, Gu F, Essa MM, Wegiel J, Kaur K, Ted Brown W et al. Brain region-specific deficit in mitochondrial electron transport chain complexes in children with autism. J Neurochem 2011; 117: 209-220.

35. Heinecke JW, Hsu FF, Crowley JR, Hazen SL, Leeuwenburgh C, Mueller DM et al. Detecting oxidative modification of biomolecules with isotope dilution mass spectrometry: sensitive and quantitative assays for oxidized amino acids in proteins and tissues. Methods Enzymol 1999; 300: 124-144

36. Gardner PR, Fridovich I. Superoxide sensitivity of the Escherichia coli aconitase. J Biol Chem 1991; 266: 19328-19333.

37. Koen AL, Goodman M. Aconitate hydratase isozymes: subcellular location, tissue distribution and possible subunit structure. Biochim Biophys Acta 1969; 191: 698-701.

38. Fridovich I. Superoxide anion radical (O2-.), superoxide dismutases, and related matters. J Biol Chem 1997; 272: 18515-18517.

39. Gardner PR, Fridovich I. Inactivation-reactivation of aconitase in Escherichia coli. A sensitive measure of superoxide radical. J Biol Chem 1992; 267: 8757-8763.

40. Melnyk S, Pogribna M, Pogribny I, Hine RJ, James SJ. A new HPLC method for the simultaneous determination of oxidized and reduced plasma aminothiols using coulometric electrochemical detection. J Nutr Biochem 1999; 10: 490-497.

41. Melnyk S, Pogribna M, Pogribny IP, James SJ. Measurement of plasma and intracellula S-adenosylmethionine and S-adenosylhomocysteine utilizing coulemetric electrochemical detection: alteration with plasma homocysteine and pyridoxal $5^{\prime}$-phosphate concentrations. Clin Chem 2000; 46: 265-272.

42. Chango A, Abdel Nour AM, Niquet C, Tessier FJ. Simultaneous determination of genomic DNA methylation and uracil misincorporation. Med Princ Pract 2009; 18: 81-84.

43. Helbock HJ, Beckman KB, Shigenaga MK, Walter PB, Woodall AA, Yeo HC et al. DNA oxidation matters: the HPLC-electrochemical detection assay of 8-oxo-deoxyguanosine and 8-oxo-guanine. Proc Natl Acad Sci USA 1998; 95: 288-293.

44. Sajdel-Sulkowska EM, Lipinski B, Windom H, Audhya T, McGinnis W. Oxidative stress in autism: cerebellar 3 nitrotyrosine levels. Am J Biochem Biotechnol 2008; 4: 73-84.

45. Liang LP, Ho YS, Patel M. Mitochondrial superoxide production in kainate-induced hippocampal damage. Neuroscience 2000; 101: 563-570.

46. Liochev SI, Fridovich I. The role of O2.- in the production of HO: in vitro and in vivo. Free Radic Biol Med 1994; 16: 29-33.

47. Vasquez-Vivar J, Kalyanaraman B, Kennedy MC. Mitochondrial aconitase is a source of hydroxyl radical. An electron spin resonance investigation. J Biol Chem 2000; 275: 14064-14069.

48. James SJ, Rose S, Melnyk S, Jernigan S, Blossom S, Pavliv 0 et al. Cellular and mitochondrial glutathione redox imbalance in lymphoblastoid cells derived from children with autism. FASEB J 2009; 23: 2374-2383.

49. Welter R, Yu L, Yu CA. The effects of nitric oxide on electron transport complexes. Arch Biochem Biophys 1996; 331: 9-14.

50. Bubber P, Hartounian V, Gibson GE, Blass JP. Abnormalities in the tricarboxylic acid (TCA) cycle in the brains of schizophrenia patients. Eur Neuropsychopharmacol 2011; 21: 254-260.

51. Prabakaran S, Swatton JE, Ryan MM, Huffaker SJ, Huang JT, Griffin JL et al. Mitochondrial dysfunction in schizophrenia: evidence for compromised brain metabolism and oxidative stress. Mol Psychiatry 2004; 9: 684-697, 643

52. Sorolla MA, Reverter-Branchat G, Tamarit J, Ferrer I, Ros J, Cabiscol E. Proteomic and oxidative stress analysis in human brain samples of Huntington disease. Free Radic Biol Med 2008; 45: 667-678

53. Liang LP, Patel M. Iron-sulfur enzyme mediated mitochondrial superoxide toxicity in experimental Parkinson's disease. J Neurochem 2004; 90: 1076-1084.

54. Pitkanen S, Robinson BH. Mitochondrial complex I deficiency leads to increased production of superoxide radicals and induction of superoxide dismutase. $J$ Clin Invest 1996; 98: 345-351. 
55. Frye RE, Rossignol DA. Mitochondrial dysfunction can connect the diverse medical symptoms associated with autism spectrum disorders. Pediatr Res 2011; 69(5 Part 2): 41R-47R

56. Rossignol DA, Frye RE. Mitochondrial dysfunction in autism spectrum disorders: a systematic review and meta-analysis. Mol Psychiatry 2012; 17: 290-314.

57. Giulivi C, Zhang YF, Omanska-Klusek A, Ross-Inta C, Wong S, Hertz-Picciotto I et al. Mitochondrial dysfunction in autism. JAMA 2010; 304: 2389-2396.

58. Mohiuddin I, Chai H, Lin PH, Lumsden AB, Yao Q, Chen C. Nitrotyrosine and chlorotyrosine: clinical significance and biological functions in the vascular system. J Surg Res 2006; 133: 143-149.

59. Bishop A, Anderson JE. NO signaling in the CNS: from the physiological to the pathological. Toxicology 2005; 208: 193-205.

60. Brown GC. Nitric oxide and mitochondrial respiration. Biochim Biophys Acta 1999; 1411: 351-369.

61. Zoroglu SS, Yurekli M, Meram I, Sogut S, Tutkun H, Yetkin O et al. Pathophysiological role of nitric oxide and adrenomedullin in autism. Cell Biochem Funct 2003; 21: 55-60.

62. Sweeten TL, Posey DJ, Shankar S, McDougle CJ. High nitric oxide production in autistic disorder: a possible role for interferon-gamma. Biol Psychiatry 2004; 55: 434-437.

63. Bolanos JP, Herrero-Mendez A, Fernandez-Fernandez S, Almeida A. Linking glycolysis with oxidative stress in neural cells: a regulatory role for nitric oxide. Biochem Soc Trans 2007; 35: 1224-1227.
64. Brown GC, Bolanos JP, Heales SJ, Clark JB. Nitric oxide produced by activated astrocytes rapidly and reversibly inhibits cellular respiration. Neurosci Lett 1995; 193: 201-204.

65. Green PS, Mendez AJ, Jacob JS, Crowley JR, Growdon W, Hyman BT et al. Neuronal expression of myeloperoxidase is increased in Alzheimer's disease. $J$ Neurochem 2004; 90: 724-733

66. Choi DK, Pennathur S, Perier C, Tieu K, Teismann P, Wu DC et al. Ablation of the inflammatory enzyme myeloperoxidase mitigates features of Parkinson's disease in mice. J Neurosci 2005; 25: 6594-6600.

67. Nagra RM, Becher B, Tourtellotte WW, Antel JP, Gold D, Paladino T et al Immunohistochemical and genetic evidence of myeloperoxidase involvement in multiple sclerosis. J Neuroimmunol 1997; 78: 97-107.

68. Pilger A, Rudiger HW. 8-Hydroxy-2'-deoxyguanosine as a marker of oxidative DNA damage related to occupational and environmental exposures. Int Arch Occup Environ Health 2006; 80: 1-15.

Translational Psychiatry is an open-access journal published by Nature Publishing Group. This work is licensed under the Creative Commons Attribution-Noncommercial-No Derivative Works 3.0 Unported License. To view a copy of this license, visit http://creativecommons.org/licenses/by-nc-nd/3.0/

Supplementary Information accompanies the paper on the Translational Psychiatry website (http://www.nature.com/tp) 